\title{
Precision laser spectroscopy of the ground state hyperfine splitting in muonic hydrogen
}

\author{
Sohtaro Kanda* \\ RIKEN Nishina Center for Accelerator-Based Science, RIKEN, Wako, Saitama 351-0198, Japan \\ E-mail: Sohtaro.kandadriken.jp \\ K. Ishida, M. Iwasaki, Y. Ma, A. Takamine, H. Ueno \\ RIKEN Nishina Center for Accelerator-Based Science, RIKEN, Wako, Saitama 351-0198, Japan
}

\author{
K. Midorikawa, N. Saito, S. Wada, M. Yumoto \\ RIKEN Center for Advanced Photonics, RIKEN, Wako, Saitama 351-0198, Japan
}

\section{S. Okada}

Atomic, Molecular, and Optical Physics Laboratory, RIKEN, Wako, Saitama 351-0198, Japan

\section{Y. Oishi, M. Sato}

High Energy Accelerator Research Organization (KEK), Tsukuba, Ibaraki 305-0801, Japan

\section{S. Aikawa}

Department of Physics, Tokyo Institute of Technology, Meguro, Tokyo 152-8551, Japan

\section{K. S. Tanaka}

Cyclotron and Radioisotope Center, Tohoku University, Sendai, Miyagi, 980-8578, Japan

\section{Y. Matsuda}

Graduate School of Arts and Sciences, The University of Tokyo, Meguro, Tokyo 153-8902, Japan

The proton is a fundamental constituent of the matter. However, it has a complicated internal structure which is difficult to be fully understood. The internal structure of the proton is described by the electronic and magnetic form factors. The charge radius of the proton is defined by these form factors and has been determined experimentally. In recent years, a significant discrepancy between independent measurements of the proton charge radius was reported. This conflict is known as "proton radius puzzle". Even though various interpretations have been proposed, no definitive solution to the problem has been found yet. In order to shed some light on the puzzle, we proposed a new experiment to determine the proton Zemach radius which is defined as a convolution of the charge distribution with the magnetic moment distribution. The proton Zemach radius can be derived from the hyperfine splitting (HFS) in the muonic hydrogen atom. We aim to perform a laser spectroscopy of the muonic hydrogen HFS with the relative uncertainty of $1 \mathrm{ppm}$ and obtain the proton Zemach radius with $1 \%$ precision.

The 19th International Workshop on Neutrinos from Accelerators-NUFACT2017

25-30 September, 2017

Uppsala University, Uppsala, Sweden

\footnotetext{
${ }^{*}$ Speaker.
} 


\section{Proton Radius Puzzle}

The proton is a fundamental constituent of the matter along with the neutron. However, its internal structure is complex because of difficulty in the quantum chromo dynamics. Elucidation of the proton internal structure is a primordial question of the fundamental physics. In order to investigate the internal structure of the proton, measurements of the charge radius and the magnetic moment distribution are essential.

The charge radius of the proton has been measured by two independent techniques; electronproton scattering and laser spectroscopy of hydrogen-like atoms. Muonic hydrogen has the Bohr radius of 200 times smaller than that of an electronic hydrogen. Therefore, a muonic hydrogen is quite sensitive to the electric and magnetic structure of the proton.

In 2010, the Lamb shift of muonic hydrogen was measured and the proton charge radius was determined at Paul Scherrer Institute (PSI) [四]. The obtained charge radius was approximately 4\% or seven standard-deviations smaller than that was obtained by the electron-proton scattering and hydrogen atom spectroscopy [0]. This conflict between the electronic and muonic measurements is referred to as" proton radius puzzle". The experimental results have been validated and reproduced in both measurements [ [3], [A, []]. Even though various interpretations were proposed to explain the discrepancy, there is no definitive solution yet.

Alternatively, the proton structure is described by the Zemach radius, which is defined as a convolution of the charge distribution with the magnetic moment distribution. Figure $\mathbb{W}$ shows the charge and Zemach radii obtained by three independent methods. In contrast to the case of the charge radius, the determination precision of the Zemach radius by the muonic hydrogen spectroscopy was insufficient to discuss the consistency between measurements. This is because the muonic result was indirectly derived from two Lamb shift frequencies via the $2 \mathrm{~S}$ hyperfine splitting. Therefore, a new direct measurement of the proton Zemach radius in a muonic system is highly desired to shed some light on this unsolved problem.
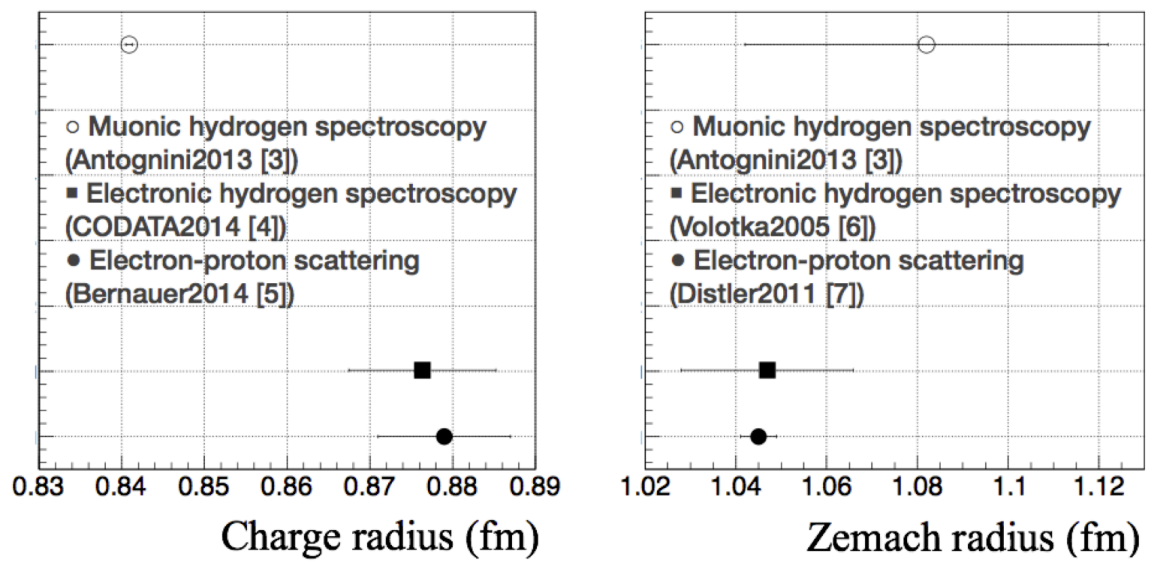

Figure 1: The proton radii obtained by the electronic and muonic measurements: (left) the charge radius

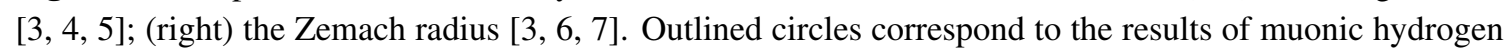
spectroscopy. Solid squares and circles correspond to the results of electronic hydrogen spectroscopy and electron-proton scattering, respectively. 


\section{Hyperfine Splitting of Muonic Hydrogen}

The hyperfine splitting (HFS) of the muonic hydrogen originates from the interaction of the magnetic moments of the muon and the proton. Theoretically, the HFS energy is described by the addition of correction terms to the Fermi energy

$$
\Delta_{\mathrm{HFS}}=E_{F}\left(1+\delta_{\mathrm{QED}}+\delta_{\text {Proton }}\right)
$$

where $E_{F}$ is the Fermi energy, $\delta_{\mathrm{QED}}$ is the higher-order electromagnetic correction, and $\delta_{\text {Proton }}$ is the correction arising from the hadronic interaction and the proton structure. In more detail, $\delta_{\text {Proton }}$ is factorized

$$
\delta_{\text {Proton }}=\delta_{\text {Rec. }}+\delta_{\text {Pol. }}+\delta_{\mathrm{HVP}}+\delta_{\text {Zemach }}
$$

where $\delta_{\mathrm{Rec}}$ is the recoil correction, $\delta_{\mathrm{Pol}}$ is the dynamical polarizability correction, $\delta_{\mathrm{HVP}}$ is the hadronic vacuum polarization term, and $\delta_{\text {Zemach }}$ is the correction arising from the finite volume of the proton. The last term is proportional to the proton Zemach radius

$$
\delta_{\mathrm{Zemach}}=-2 \alpha m_{\mu p} R_{Z}
$$

where $\alpha$ is the fine structure constant, $m_{\mu p}$ is the reduced mass of the muonic hydrogen, and $R_{Z}$ is the Zemach radius. The most recent theoretical calculation predicts $\Delta_{\mathrm{HFS}}=182.638(62) \mathrm{meV}$ and $\delta_{\text {Zemach }}=-1.362 \mathrm{meV}[\mathrm{l}]$ where the error comes mostly from present experimental uncertainties of the proton form factors. We aim to determine the proton Zemach radius via a measurement of the muonic hydrogen HFS. A measurement of the muonic hydrogen HFS with the relative uncertainty of $2 \mathrm{ppm}$ derives the proton Zemach radius with $1 \%$ precision. The determination precision is limited by the theoretical uncertainty of the proton polarizability correction [Q]].

\section{Experimental Overview}

Figure $\square$ illustrates a schematic of the experimental apparatus for the muonic hydrogen HFS spectroscopy. The experiment will be performed at J-PARC MLF. Negative pulsed muon beam irradiates a gas chamber filled with cryogenic hydrogen gas. The gas temperature and pressure are $20 \mathrm{~K}$ and $6 \mathrm{hPa}$, respectively. The target density is $0.1 \%$ of the liquid hydrogen density (LHD). The cryogenic temperature and the low density of the target are of importance to suppress the Doppler broadening effect, muonic molecule formation, and hyperfine de-excitation due to the atomic collisions [ए]]. An injected muon loses its kinetic energy and forms a muonic hydrogen. Two highly reflective mirrors are placed inside the gas chamber. These two mirrors are arranged to be facing each other in order to constitute a multi-pass cell for laser light confinement. An intense pulsed mid-infrared laser light is injected to the multi-pass cell and the HFS transition of the muonic hydrogen is induced. The details of the transition laser are described in the following section. The state transition causes a muon spin flip as a function of time. An electron is emitted by parity violating decay of muon and its emission angle is correlated to the muon spin direction. Spectroscopy of the muonic hydrogen HFS is performed by a measurement of laser frequency dependence of the number of decay electrons from muonic hydrogen decays. Electrons are detected by a segmented scintillation counter. The detectors are placed so as to surround the target gas 
chamber. The gas chamber is made of tungsten in order to reduce background events arising from muons which stopped on the chamber wall. A muon stopped in the heavy material decays with short lifetime due to the large cross section of the nuclear capture process.

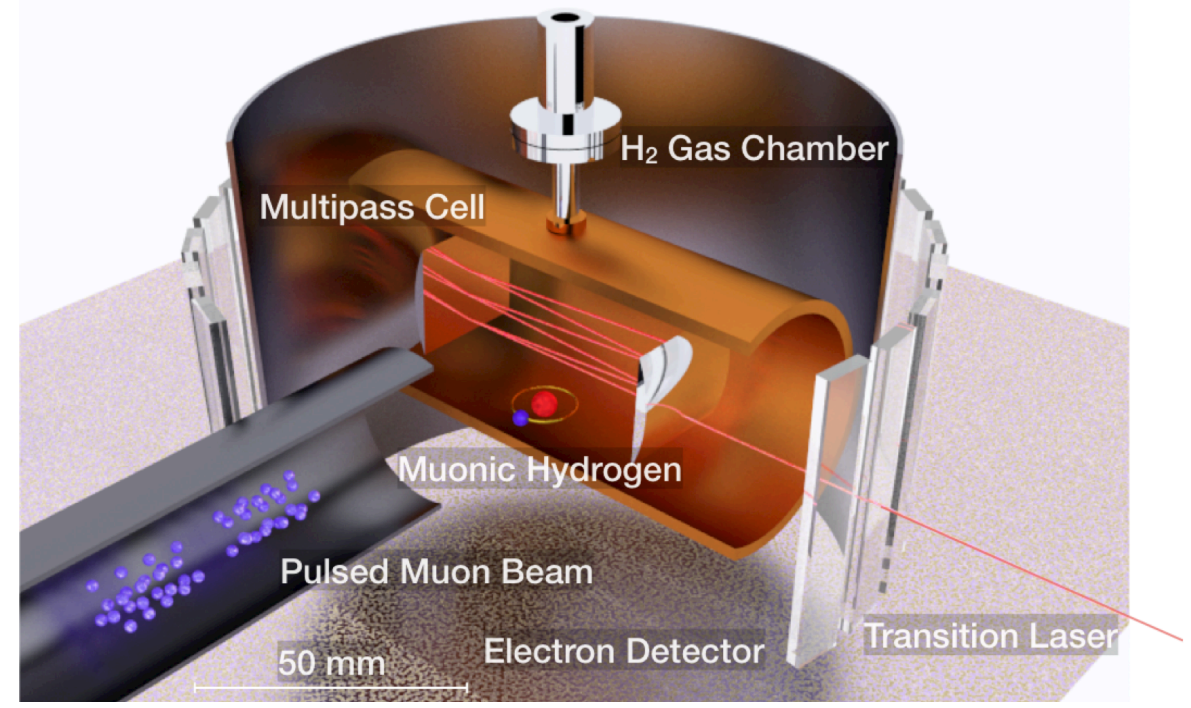

Figure 2: Experimental schematic. Pulsed muon beam irradiates the $\mathrm{H}_{2}$ gas target. Muon is captured by the Coulomb field of the proton and forms a muonic hydrogen atom. The hyperfine transition between the spin singlet state and the triplet states is induced by a mid-infrared laser light. Electrons from muonic hydrogen decay are detected by the electron counter placed around the gas chamber.

\section{Laser System}

The hyperfine splitting in the ground-state muonic hydrogen is $183 \mathrm{meV}$ and a coherent light having the wavelength of $6.8 \mu \mathrm{s}$ is required. This HFS excitation is a magnetic dipole transition and suppressed by the selection rule. Therefore, a high pulse energy is of necessity for the HFS transition compared to the Lamb shift case. The transition probability $P$ obeys an equation $P=$ $2 \times 10^{-5} E / S / \sqrt{T}$ where $E$ is the laser pulse energy, $S$ is the cross-section of the laser beam, and $T$ is the temperature [W]. The reflection mirrors multiply the transition probability by the number of laser light reflection $N$. The experimental design parameters are $E=20 \mathrm{~mJ}, S=4 \mathrm{~cm}^{2}, T=20 \mathrm{~K}$ and $N=2000$. The number of reflection $N=2000$ corresponds to the mirror reflective index of $99.95 \%$. Under these conditions, the averaged muon spin polarization was estimated to be $2 \%$.

The frequency tunability should be greater than the theoretical uncertainty of the muonic hydrogen HFS. The uncertainty of the theoretical prediction is $62 \mu \mathrm{eV}$ and it corresponds to the frequency tunability of $15 \mathrm{GHz}$. The spectral linewidth of the laser should be comparable to the linewidth of the HFS resonance line-shape. The width of the resonance is dominated by the Doppler broadening effect because the natural linewidth is quite narrow. The Doppler broadening effect is estimated to be $57 \mathrm{MHz}$ at the target temperature of $20 \mathrm{~K}$. A narrow pulse timing width is desirable to obtain a high conversion efficiency at the frequency conversion process which is mentioned later. The pulse width of $150 \mathrm{~ns}$ is required for sufficient pulse energy. Laser injections are to be synchronized with the pulsed muon beam arrival. At J-PARC MLF, the beam repetition frequency

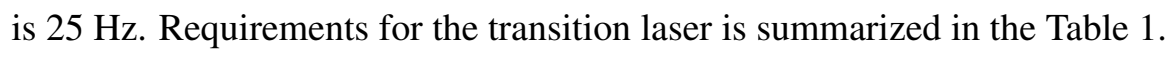


Table 1: Specification requirements for the transition laser.

\begin{tabular}{ccc}
\hline Specification & Quantity & Relevant Experimental Parameter \\
\hline Wavelength & $6.789 \mu \mathrm{m}(44 \mathrm{THz})$ & HFS energy \\
Wavelength tunability & $\geq 2.3 \mathrm{~nm}(15 \mathrm{GHz})$ & HFS uncertainty \\
Spectral linewidth & $\leq 0.02 \mathrm{~nm}(100 \mathrm{MHz})$ & Doppler broadening \\
Pulse energy & $\geq 20 \mathrm{~mJ}$ & Transition probability \\
Pulse width & $\leq 150 \mathrm{~ns}$ & Light conversion efficiency \\
Pulse repetition & $25 \mathrm{~Hz}$ & Muon beam repetition \\
Polarization & Circular & Selective excitation of the triplet state
\end{tabular}

The coherent light having the wavelength of $6.8 \mu \mathrm{m}$ is generated by an optical parametric oscillator (OPO) using a $\mathrm{ZnGeP}_{2}$ (ZGP) nonlinear optical crystal. The ZGP-OPO is pumped with a $\mathrm{Tm}^{3+}$ and $\mathrm{Ho}^{3+}$ co-doped YAG ceramic laser. A quantum cascade laser (QCL) is employed as a narrowband seeder for the OPO. The output beam of the OPO is amplified by a ZGP optical parametric amplifier (OPA). Figure [ ] depicts a diagram of the laser system.

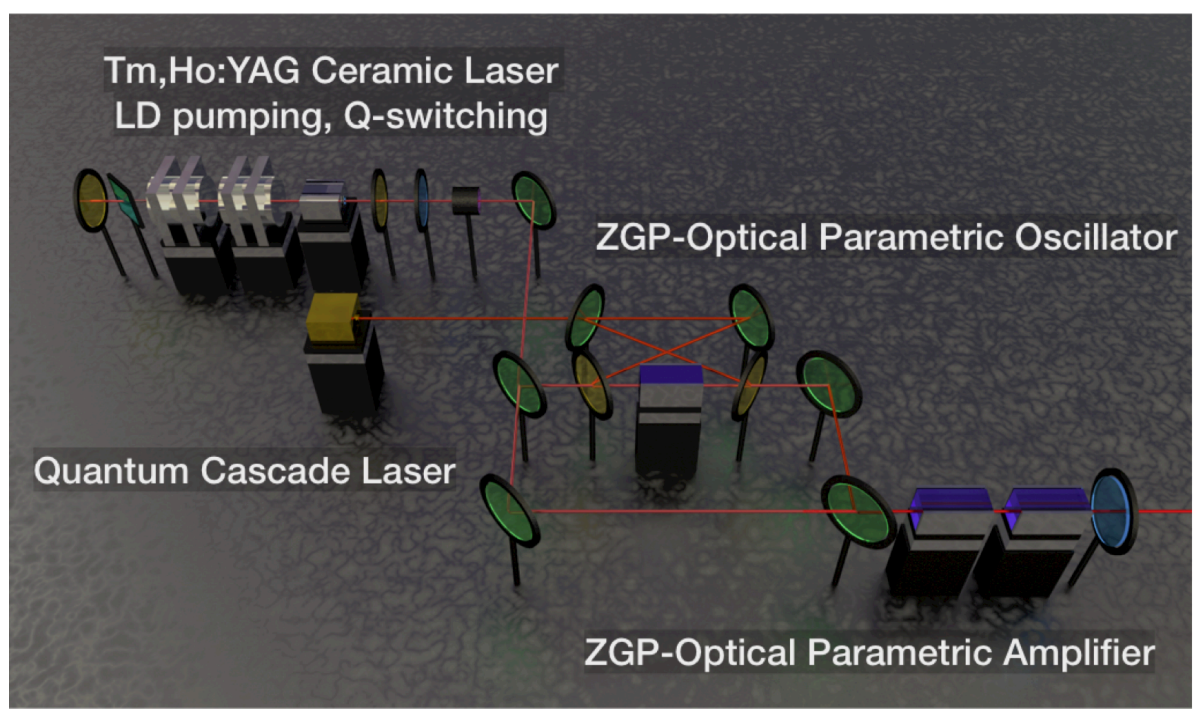

Figure 3: Laser system for the high pulse energy $6.8 \mu \mathrm{m}$ coherent light source. The Tm,Ho:YAG ceramic laser is pumped by the laser diodes and it oscillates at $2.09 \mu \mathrm{m}$. The output beams of the Tm,Ho:YAG laser and the QCL are pump and seed beam for the ZGP-OPO, respectively. The OPO oscillates at $6.8 \mu \mathrm{m}$ and it is amplified by the ZGP-OPA. The output beam of the OPA is circularly polarized using a quarter-waveplate.

An OPO is a device which coverts an incident photon to two photons with different frequencies. The conversion follows the energy conservation, i.e., the summation of the output frequencies equals to the input frequency. Two output beams are termed a signal light and an idler light. The convention prescribes that the idler light has a longer wavelength. Under the $2.09 \mu \mathrm{m}$ pumped, type-I ZGP-OPO with the phase matching angle of 52.5 degree, the wavelengths of the signal and the idler lights are $3.0 \mu \mathrm{m}$ and $6.8 \mu \mathrm{m}$, respectively. The optical damage threshold of ZGP crystal is $2 \mathrm{~J} / \mathrm{cm}^{2}$ [ए2]. The radius of the pumping beam is $1 \mathrm{~mm}$ and the maximum pumping energy without crystal damage was estimated to be $60 \mathrm{~mJ}$. For stable operation of the laser system, the pumping energy is set $40 \mathrm{~mJ}$. The conversion efficiency of $12 \%$ was reported at the idler wavelength of 6.6 
$\mu \mathrm{m}$ [[13]. A similar level of the conversion efficiency is expected for our experimental case.

For a precision spectroscopy of the muonic hydrogen HFS, a narrowband $6.8 \mu \mathrm{m}$ coherent light is required. Narrowing of the spectral linewidth is expected under the injection-seeded OPO with a narrowband seeder [14]]. The QCL is adopted as a seeder for the ZGP-OPO and an expected spectral linewidth is less than $100 \mathrm{MHz}$. The idler light generated by the OPO is amplified using the ZGP-OPA [ㅍ]. The OPA is pumped with the Tm,Ho:YAG ceramic laser. A factor of two amplification is expected under the ZGP-OPA. Then, the pulse energy of $10 \mathrm{~mJ}$ or higher is expected after OPA. In the spectroscopy experiment, two sets of the laser system will be employed for the total energy of $20 \mathrm{~mJ}$. The quarter waveplate is placed after the OPA to obtain a circularly polarized beam. Only the state transition with $\Delta F_{z}=+1$ contributes to the muon spin asymmetry where $F_{z}$ is the z-component of total angular momentum of the muonic hydrogen. The generated mid-infrared beam is delivered to the multi-pass cell which is installed inside the hydrogen gas target. The cell consists of two highly reflective mirrors with dielectric multi-layer coating.

\section{Feasibility of the Experiment}

The experiment was proposed to be performed at the D2 beamline of MUSE facility at JPARC MLF. The muon beam intensity of $1 \times 10^{6} \mu^{-} / \mathrm{s}$ is expected at the beam momentum of 40 $\mathrm{MeV} / \mathrm{c}$ and the accelerator operation power of $1 \mathrm{MW}$. The beam has a pulsed timing structure and its repetition frequency is $25 \mathrm{~Hz}$. The full width at half maximum of a beam pulse is $100 \mathrm{~ns}$.

For a case of the target density of $0.1 \%$ LHD, approximately $0.05 \%$ of the incident muons are stopped in the multi-pass cell. Other muons are absorbed by heavy nucleus in the surrounding apparatuses. Figure $\mathbb{t}$ shows a simulated time spectrum of electron emission from muon decays.

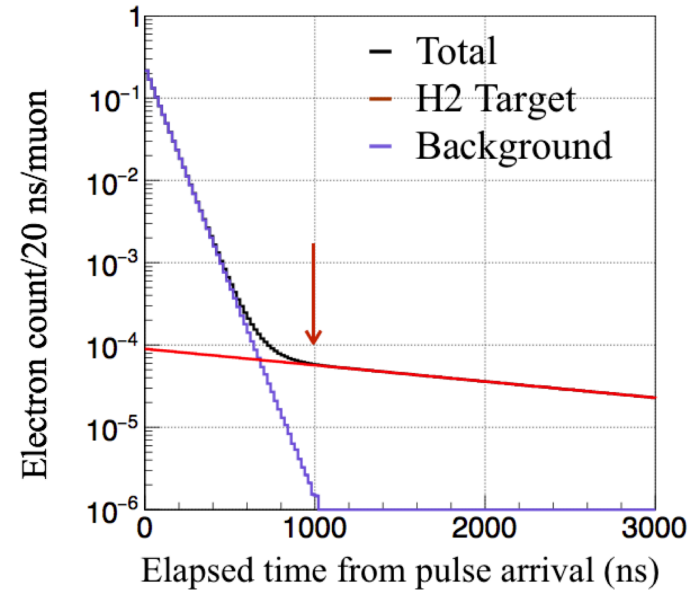

Figure 4: Simulated time spectrum of muon decay. Red and blue histograms correspond to the muons stopped in the hydrogen target, other apparatuses. Black one corresponds to total stopped muons. Red arrow indicates laser injection timing.

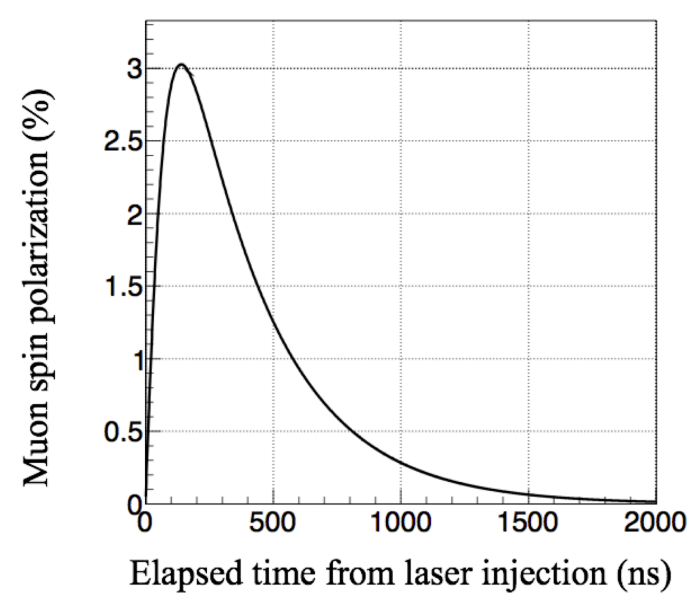

Figure 5: Calculated muon spin polarization arising from the hyperfine transition of muonic hydrogen. The laser pulse energy of $20 \mathrm{~mJ}$, the mirror reflectivity of $99.95 \%$, and the hyperfine quenching rate of 20 $\mathrm{MHz}$ were assumed.

The transition laser light is injected $1 \mu \mathrm{s}$ after the muon pulse arrival and repetitively reflected between two mirrors in the multi-pass cell. Figure $\$$ shows a calculated muon spin polarization. 
Light attenuation by reflection and polarization extinction due to the collisional quenching were considered. From a view point of the averaged polarization, an optimal width of measurement timing window was set $700 \mathrm{~ns}$. The averaged muon spin polarization of $2 \%$ is expected.

The number of muonic hydrogen was estimated to be 500 per second and 85 decay electrons are expected in the timing window aforementioned. The electron acceptance of the electron detector is $25 \%$ and the angular asymmetry of the decay electrons is $33 \%$. Hence, the number of expected spin flip signal is 0.14 per second. When a frequency of the transition laser is set the HFS frequency, a three-hours of measurement gives three standard deviations of significance. If the HFS resonance exists in the range of theoretical prediction, the resonance can be observed by a frequency scan measurement at 150 data points with the interval of $100 \mathrm{MHz}$. After the discovery of the HFS resonance, a precision spectroscopy of the resonance line-shape will be performed to determine the HFS frequency with the uncertainty of $2 \mathrm{ppm}$. It requires a sixteen-hours of measurement at twelve data points. In total, a month of measurement period is necessary. This requirement is comparable with other fundamental physics projects proposed to the J-PARC MLF MUSE.

\section{Summary}

The proton radius puzzle is one of the most important unsolved problem in sub-atomic physics. For a deeper understanding of the issue, a new measurement of the ground-state hyperfine splitting in muonic hydrogen atom was proposed. The hyperfine transition is occurred by an intense mid-infrared laser. The hyperfine excited state is detected by a measurement of decay electron angular asymmetry which depends on the muon spin polarization arising from the state transition. In order to perform the experiment with high significance, development of an intense and narrowband pulsed mid-infrared light source is essential. A novel scheme of all-solid laser system was designed for the experiment. Technological development of the laser system is in progress and the experiment is feasible with the high-intensity pulsed muon beam at J-PARC MLF MUSE.

\section{References}

[1] R. Pohl et al., Nature 466, 213 (2010).

[2] P. J. Mohr, B. N. Taylor, and D. B. Newell, Rev. Mod. Phys. 84, 1527 (2012).

[3] A. Antognini et al., Science 339, 417 (2013).

[4] P. J. Mohr, D. B. Newell, and B. N. Taylor, Rev. Mod. Phys. 88, 035009 (2016).

[5] J. C. Bernauer et al., Phys. Rev. C 90, 015206 (2014).

[6] A. V. Volotka et al., Eur. Phys. J. D 33, 23 (2005).

[7] M. O. Distler et al., Phys. Lett. B 696, 343 (2011).

[8] R. N. Faustov, A. P. Martynenko, J. Exp. Theor. Phys. 98, 39 (2004).

[9] A. Dupays et al., Phys. Rev. A 68, 052503 (2003).

[10] J. S. Cohen, Phys. Rev. A 43, 4668 (1991).

[11] A. Adamczak et al., Nucl. Instrum. Meth. B 281, 72 (2012), private communication with D. Bakalov.

[12] K. T. Zawilski et al., J. Opt. Sci. Am. B 23, 11 (2006).

[13] K. L. Vodopyanov et al., Optics Lett. 25, 11, 841 (2000).

[14] H. R. Lee et al., ASSP (OSA), MC1 (2006).

[15] M. W. Haakestad et al., Optics Lett. 16, 18, 14263 (2008). 